\title{
Spectral analysis of high-harmonic coherent acoustic phonons in piezoelectric semiconductor multiple quantum wells
}

\author{
Gia-Wei Chern, Kung-Hsuan Lin, Yue-Kai Huang, and Chi-Kuang Sun* \\ Graduate Institute of Electro-Optical Engineering, National Taiwan University, Taipei 10617, Taiwan, Republic of China
}

(Received 2 January 2003; published 19 March 2003)

\begin{abstract}
Multiple harmonic components have been observed in the Fourier spectra of the coherent acoustic phonon oscillations in InGaN/GaN multiple quantum wells (MQW's). The dependence of the relative magnitudes of the first two Fourier components on the sample geometry was analyzed by conducting time-resolved pumpprobe measurements on MQW samples with different well-to-barrier ratios. The results were also compared with the recently proposed loaded-string model [Phys. Rev. B 64, 235316 (2001)], and an overall agreement is obtained which validates the picture of string oscillation with inhomogeneous loadings. We also demonstrated manipulation of the spectral components using the coherent optical control technique. Our demonstration can be regarded as a first step to the realization of wave-form synthesis of nanoacoustic waves.
\end{abstract}

DOI: 10.1103/PhysRevB.67.121303 PACS number(s): 78.47. + p, 62.25. $+\mathrm{g}$, 43.35. $+\mathrm{d}, 63.22 .+\mathrm{m}$

Following recent advances in femtosecond lasers and ultrafast spectroscopy technology, generation and control of acoustic phonons with a high degree of spatial and temporal coherence at the teraherz $(\mathrm{THz})$ frequencies have been demonstrated in bulk solids, ${ }^{1-4}$ semiconductor superlattices, ${ }^{5,6}$ quantum dots, ${ }^{7}$ metallic nanoparticles, ${ }^{8}$ and a number of other materials. The progress in coherent acoustic-phonon generation has opened possibilities for research in fundamental phonon physics ${ }^{9}$ and material characterization. ${ }^{10}$ With the ability to generate intense coherent acoustic waves, many potential applications can be exploited, for example, phonon control of electron transport in quantum heterostructures ${ }^{11}$ and phonon control of the ionic motion, ${ }^{12}$ etc. Recently, we had reported large coherent longitudinal-acoustic (LA) phonon oscillations with piezoelectric InGaN/GaN multiple quantum wells (MQW's). ${ }^{13,14}$ Coherent control within a few oscillation cycles was also demonstrated in this system. ${ }^{15,16}$ Such nitride-based piezoelectric MQW structures acting as coherent nanowavelength acoustic sources opens a new passage to phonon engineering. Furthermore, the propagating nature of coherent acoustic phonons makes it possible to realize the analogy of laser, a saser. ${ }^{17}$ The observed large oscillation amplitude renders this nitride-based MQW a possible candidate for constructing a phonon laser.

Contrary to its optical-phonon counterpart, the characteristic frequency of the coherent acoustic phonons is mostly determined by the length scale of the underlying heterostructures. Through a linear dispersion relation, the observed temporal oscillation reflects the spatial spectrum of the heterostructure. In most cases, there also exist non-negligible zonefolding phenomena, for example, in GaAs/InAs superlattices. ${ }^{6}$ As a result, coherent acoustic phonons usually have multiple spectral components. By utilizing coherent control technique, it is possible to manipulate the spectral composition of a phonon pulse, and therefore, synthesize its temporal wave form. ${ }^{18,19}$ For example, Bartels et al. have demonstrated the coherent control of zone-folded acoustic lattice vibrations in GaAs/AlAs superlattices by time sequential excitation with femtosecond pulses. In their experiments, they silenced the first back-folded mode near $q=0$, while selectively enhancing the coherent amplitude of higher-order back-folded modes. ${ }^{18}$
In the present Rapid Communication, we report the spectral analysis of the coherent acoustic phonons in the InGaN/ GaN MQW's. Higher harmonic oscillations (up to the order of 3) were also observed from the spectral analysis. By conducting pump-probe measurements on different MQW samples, we studied the dependence of the spectral components of acoustic phonons on MQW duty ratios (defined as the ratio of well thickness $L_{w}$ to the MQW period $L$ ). The experimental results are compared with the loaded-string model. ${ }^{20,21}$ Finally, similar to previous coherent control experiments, ${ }^{18,19}$ second-harmonic oscillation was enhanced by injecting another control pulse, while the fundamental oscillation was suppressed due to destructive interference.

We performed our measurements using standard femtosecond transmission pump-probe technique based on a Kerrlens mode-locked Ti: sapphire laser. The laser output pulses were frequency doubled using a beta barium borate crystal to reach the band-gap energy of the InGaN/GaN MQW's. The frequency-doubled pulses had a pulse width of $180 \mathrm{fs}$ at a wavelength of $390 \mathrm{~nm}$ measured by a two-photonabsorption-type pump-probe autocorrelation in a bulk GaN thin film. ${ }^{14,15}$ Because of the large piezoelectric constants along the [0001] orientation group III nitrides, a straininduced piezoelectric field on the order of $\mathrm{MV} / \mathrm{cm}$ is expected. This large piezoelectric field is also important for the generation of coherent acoustic phonons. ${ }^{14,20}$ The widths of barrier and well are in the range of 10-130 $\AA$, depending on samples. The In composition in the MQW's was determined to be $10 \%$. The MQW samples consist of 14 periods. $^{22}$

Figure 1(a) shows the measured differential transmission change at a wavelength of $390 \mathrm{~nm}$ after the same wavelength femtosecond pulse excitation in a 14 period InGaN MQW. The well and barrier widths of the MQW are 2.2 and $7 \mathrm{~nm}$, respectively. All the quantum wells have the same well and barrier widths. After zero time delay, a clear transmission oscillation due to the induced coherent acoustic phonons can be observed. The observed oscillation period is $1.27 \mathrm{ps}$, corresponding to an acoustic frequency of $0.8 \mathrm{THz}$. With an acoustic wavelength the same as the quantum well period, $9.2 \mathrm{~nm}$, a longitudinal acoustic velocity of $7200 \mathrm{~m} / \mathrm{s}$ in the MQW structure can be obtained. ${ }^{14}$ Due to the highly asym- 

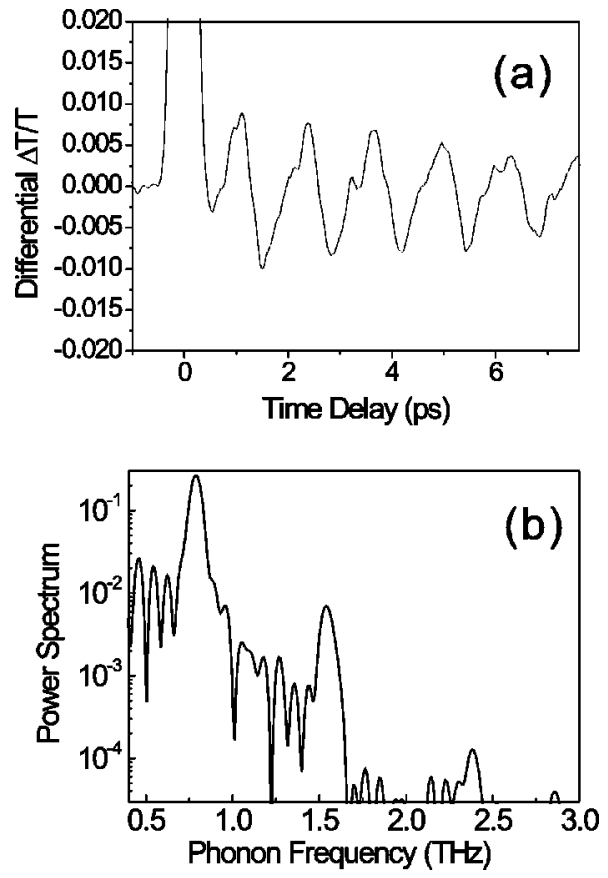

FIG. 1. (a) Measured differential transmission change versus probe delay for a $22 \AA / 70 \AA$ InGaN/GaN MQW. The modulated transmission change with a period of 1.27 ps reflects the photoinduced coherent acoustic phonon oscillation. The laser wavelength was $390 \mathrm{~nm}$. (b) Fourier power spectrum of the temporal trace shown in (a). Acoustic frequency components of $0.8,1.6$, and 2.4 $\mathrm{THz}$ can be observed with corresponding acoustic wavelengths of 9.2, 4.6, and $3.1 \mathrm{~nm}$.

metric well-to-barrier ratio (2.2 versus 7.0), higher harmonic oscillations were expected according to the loaded-string model. ${ }^{20,21}$

Figure 1(b) shows the Fourier power spectrum of the temporal trace shown in Fig. 1(a). One sharp peak at $0.8 \mathrm{THz}$ can be seen in the figure, which corresponds to the fundamental oscillation frequency of inverse $1.27 \mathrm{ps}$ in the temporal trace [Fig. 1(a)]. There are also smaller peaks at $1.6 \mathrm{THz}$ (4.6-nm acoustic wavelength) and $2.4 \mathrm{THz}$ (3.1-nm acoustic wavelength) corresponding to the second- and thirdharmonic oscillations in the temporal trace shown in Fig. 1(a). It is worth noting that there is negligible splitting of oscillation frequencies caused by zone-folding effect. This is due to the small difference of the elastic constants between well and barrier materials. The splitting is estimated to be less than $1 \%$ using available sound velocities.

In order to investigate the second-harmonic component of the generated coherent acoustic phonons, we have to look into the generation process. Recently, Sanders et al. developed a microscopic theory for the photoexcitation of carriers and subsequent generation of coherent phonons in the MQW's. ${ }^{20}$ Under typical experimental conditions, they showed that the microscopic theory could be simplified and mapped onto a loaded-string problem that can be easily solved and understood. ${ }^{20}$ On the other hand, based on piezoelectric constitution relations, continuum elastic mechanics, and Maxwell's equations, we also developed a macroscopic theory for the generation and propagation of coherent phonons in such MQW structures. ${ }^{21}$ The eigenmodes are found to obey the loaded-string equation. The generation mechanism can be understood as follows. ${ }^{14,20}$ Due to large piezoelectric field in the well, the photoexcited electrons and holes are spatially separated, resulting in a periodic space charge density within the MQW. This charge density partially screens the piezoelectric field and impulsively changes the mechanical equilibrium state to an inhomogeneous one through piezoelectric and deformation potential couplings. As a result, the lattice relaxes to this new inhomogeneous equilibrium and thereby initiates the coherent acoustic oscillations.

Let $\mathbf{u}=u \hat{\mathbf{z}}$ be the displacement field, here $z$ direction is chosen to be along the crystal $c$ axis, which is the case of our samples discussed in this Rapid Communication. The loaded string equation for $u$ is ${ }^{20,21}$

$$
\rho_{0} \frac{\partial^{2} u}{\partial t^{2}}-\Gamma \frac{\partial^{2} u}{\partial z^{2}}=-\frac{e_{33}}{\varepsilon_{3}} \rho_{s c}+\sum_{\nu=e, h} d_{\nu 3} \frac{\partial \rho_{\nu}}{\partial z}=f_{\text {piezo }}+f_{\text {def }},
$$

where $\Gamma=C_{33}+e_{33}^{2} / \varepsilon_{3}$ is the effective elastic module along [0001] direction, $C_{33}, \varepsilon_{3}$, and $e_{33}$ are the stiffness, dielectric, and piezoelectric tensor elements in the $z$ direction. $\rho_{s c}$ $=|e|\left(\rho_{e}-\rho_{h}\right)$ is the space charge density, and $\rho_{\nu}$ is the number density of species $\nu(\nu=e, h) . d_{\nu 3}$ is the deformation potential coupling constant. The sound velocity is given by $C_{s}=\sqrt{\Gamma / \rho_{0}}$. The contracted index convention is used for the tensors. We also introduce two driving forces in Eq. (1): $f_{\text {piezo }}$ and $f_{\text {def }}$, corresponding to piezoelectric and deformation potential couplings. The resulting strain is given by $s$ $=\partial u / \partial z$ and the electric field is $E=E_{s c}-\left(e_{33} / \varepsilon_{3}\right) s .{ }^{21}$ Here the space charge field satisfies $\varepsilon_{3} \partial E_{s c} / \partial z=\rho_{s c}$. The Fourier component of strain $\tilde{s}$ then satisfies the well-known simple harmonic-oscillator equation ${ }^{21,23}$

$$
\left(\frac{\partial^{2}}{\partial t^{2}}+\omega_{q}^{2}\right) \tilde{s}(q, t)=\frac{i q}{\rho_{0}} \widetilde{f}(q, t) .
$$

Here $\widetilde{f}$ is the Fourier transform of the driving force and the angular frequency $\omega_{q}=C_{s} q$. Assuming the pump pulse has a width much less than the oscillation period, the solution of $\tilde{s}$ is given by ${ }^{21,23}$

$$
\tilde{s}(q, t)=\left[i \widetilde{f}(q) / \rho_{0} C_{s}^{2} q\right]\left[1-\widetilde{I}\left(\omega_{q}\right) \cos \left(\omega_{q} t\right)\right] .
$$

Here $\tilde{f}(q)=\widetilde{f}\left(q, t \gg \tau_{p}\right)$ is the steady-state value and $\tau_{p}$ is the laser pulsewidth. There are two terms on the right-hand side of Eq. (3). The first term corresponds to the steady state of the carrier-induced strain field $\tilde{s}_{0}(q)=i \widetilde{f}(q) / \rho_{0} C_{s}^{2} q$. The second term corresponds to the oscillating part of the strain field, i.e., the coherent acoustic waves. The magnitude of the coherent acoustic wave is also proportional to the steadystate strain component $\tilde{s}_{0}(q)$ and is reduced by the pulse spectrum factor $\widetilde{I}\left(\omega_{q}\right) .{ }^{21,23}$ The strain field $\tilde{s}$ has the same periodicity as the source term $\widetilde{f}$, which is a periodic function with a period equal to $L$. Their Fourier components have peaks at $q=m q_{0}$ with $q_{0}=2 \pi / L$ and $m$ integers. As a result, 


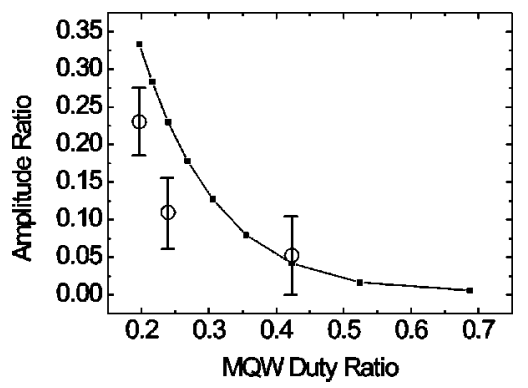

FIG. 2. Amplitude ratio of second harmonic to fundamental oscillations $\left|\tilde{s}\left(2 q_{0}\right)\right| /\left|\tilde{s}\left(q_{0}\right)\right|$ versus duty ratio of MQW, $L_{w} / L$. The InGaN/GaN MQWs considered here have a fixed well thickness $L_{w}=22 \AA$. The solid line is calculated by using the loaded string model. The open circles are measured results, corresponding to barrier thicknesses of 30, 70, and $90 \AA$.

the characteristic oscillating angular frequencies are $m \omega_{0}$ $=m C_{s} q_{0}$. The relative magnitudes of the Fourier components depend on the structure of the MQW.

In Fig. 2, we show the relative oscillating amplitude of second harmonic $\tilde{s}\left(2 q_{0}\right)$ to $\tilde{s}\left(q_{0}\right)$ (the left vertical axis) versus the MQW duty ratio $L_{w} / L$ (solid line). In the calculation, the well thickness was kept $22 \AA$, and the barrier thickness was varied from 10 to $90 \AA$. Here we assume a sech ${ }^{2}$ intensity profile (autocorrelation full width at half maximum equal to $255 \mathrm{fs}$ ) for the calculation of the spectrum factor $\widetilde{I}\left(\omega_{q}\right)$. An increase in the second-harmonic component with a more asymmetric well-barrier width ratio can be observed. Also shown in Fig. 2 (open circles) are the observed Fourier component ratios $\left(2 \omega_{0}\right.$ to $\left.\omega_{0}\right)$ of the measured $\Delta T / T$ corresponding to traces from different samples with different well/ barrier width ratios of $22 \AA / 30 \AA, 22 \AA / 70 \AA$, and $22 \AA / 90 \AA$ InGaN/GaN MQWs. Since the observed phonon-induced transmission modulation $\Delta T / T$ is through the quantum confined Franz-Keldysh (QCFK) effect, ${ }^{24}$ it depends on the relative overlapping of strain field with the electron and hole envelope wave functions. The modulation efficiencies of coherent oscillations with different spatial Fourier components are also different. It is expected that the first-order component would have a larger QCFK effect due to a greater overlap with the envelope functions. It can be seen from Fig. 2 that the observed modulation ratio is slightly lower than the calculated strain amplitude ratio. An overall agreement of the measured values with expected values from the loaded-string model is obtained.

In order to selectively enhance the second-harmonic component of the initiated coherent acoustic phonons, we also apply coherent optical control to perform the coherent cancellation of the fundamental and third-harmonic components with simultaneous enhancement of the second-harmonic component. We have previously demonstrated coherent control of acoustic phonon oscillations in InGaN/GaN MQW's for coherent manipulation, including magnitude and phase, of the initial fundamental oscillation. ${ }^{15,16}$ The fact that the LA phonon oscillation in InGaN/GaN MQW has multiple harmonic components is a special feature compared to others. By applying the coherent control technique to the generated acoustic-phonon oscillations, we are able to selec-
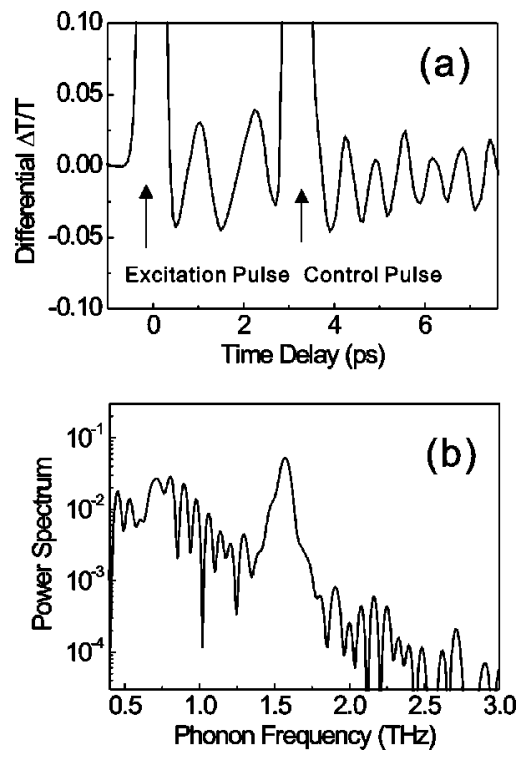

FIG. 3. (a) Measured differentiated transmission changes versus probe delay with a control pulse at 2.5 fundamental oscillation cycles after the first pump pulse. Second-harmonic oscillation with a period of 0.63 ps can be clearly observed after applying coherent control with a control pulse. (b) Fourier power spectra of the temporal traces shown in (a) after the incidence of the control pulse. The enhancement of the second-harmonic component is evident.

tively enhance the second-harmonic component while suppressing the fundamental and third-harmonic ones. This was done by applying another femtosecond uv pulse (called control pulse) into the pump region at 3.18-ps time delay, which is equal to 2.5 fundamental oscillation cycles, 5.0 second-harmonic oscillation cycles, or 7.5 third-harmonic oscillation cycles. This control pulse thus generated another coherent acoustic-phonon oscillation of which fundamental and third-harmonic oscillations destructively interfered with the original ones and second-harmonic component constructively interfered with the original second-harmonic component.

Figure 3(a) shows the measured differential transmission change induced by the pump pulse and a 3.18-ps delayed control pulse. In the coherent control experiment, after initiation of the oscillation, an uv control pulse was directed into the same area to perform the oscillation manipulation with time delay controlled by a $0.1-\mu \mathrm{m}$ stepping stage. In order to have a maximum spatial overlap between the pump and control pulses, the incident angles of these two pulses were carefully tuned so that they were identical. A clear second harmonic oscillation with an oscillation period of 0.63 ps, half of the fundamental oscillation period, can be observed after applying the control pulse at 3.18 ps. Figure 3(b) shows the Fourier intensity spectra of the trace after incidence of the control pulses. Consistent with time-domain observations, the magnitude of the second-harmonic oscillation was significantly enhanced relative to the fundamental one. It is also interesting to notice the suppression of the weak thirdharmonic oscillation, since we are also performing destructive interference for the third-harmonic oscillation.

In summary, we have presented experimental spectral 
analysis of the coherent LA phonon oscillations in InGaN/ GaN MQW's. By conducting time-resolved pump-probe measurements on MQW samples with different barrier thickness, we studied the dependence of the spectral components on the sample geometry. The results were also compared to a loaded-string model and an overall agreement was obtained. Simultaneously, cancellation of the fundamental oscillation and enhancement of the second harmonic was also demonstrated through optical coherent controls. Our demonstration indicates the possibility for nanoacoustic wave-form synthesis. Different acoustic frequencies can be predesigned to be embedded in the MQWs or superlattices with different period widths and well-to-barrier width ratios. Acoustic waveform synthesis up to a level of nanometers should then be possible by coherently controlling the phases and magnitudes of different Fourier acoustic wavelength components. This simple realization of spectral manipulation is a first step for complicated wave-form synthesis, which might lead to potential applications including material characterizations, time-domain phonon spectroscopy, nanoacoustic imaging, and even nanononlinear acoustics.

The InGaN MQW sample was kindly provided by S. P. DenBaars. The authors would like to acknowledge stimulating scientific discussions with C. J. Stanton, G. D. Sanders, and F. Vallée. This work was sponsored by National Science Council of Taiwan, R.O.C. under Grants Nos. 912112-M-002-050 and NSC91-2215-E-002-021. K.-H. Lin would like to thank SiS Education Foundation for financial assistance.
*Corresponding author. Email address: sun@cc.ee.ntu.edu.tw

${ }^{1}$ C. Thomsen, J. Strait, Z. Vardeny, H.J. Maris, J. Tauc, and J.J. Hauser, Phys. Rev. Lett. 53, 989 (1984).

${ }^{2}$ K.A. Nelson, D.R. Lutz, M.D. Fayer, and L. Madison, Phys. Rev. B 24, 3261 (1981).

${ }^{3}$ Y.-K. Huang, G.-W. Chern, C.-K. Sun, Y. Smorchkova, S. Keller, U. Mishra, and S.P. DenBaars, Appl. Phys. Lett. 79, 3361 (2001).

${ }^{4}$ M.D. Cummings and A.Y. Elezzabi, Appl. Phys. Lett. 79, 770 (2001).

${ }^{5}$ A. Yamamoto, T. Mishina, Y. Masumoto, and M. Nakayama, Phys. Rev. Lett. 73, 740 (1994).

${ }^{6}$ A. Bartels, T. Dekorsy, H. Kurz, and K. Köhler, Phys. Rev. Lett. 82, 1044 (1999).

${ }^{7}$ T.D. Krauss and F.W. Wise, Phys. Rev. Lett. 79, 5102 (1997).

${ }^{8}$ M. Nisoli, S. De Silvestri, A. Cavalleri, A.M. Malvezzi, A. Stella, G. Lanzani, P. Cheyssac, and R. Kofman, Phys. Rev. B 55, R13 424 (1997).

${ }^{9}$ H.-Y. Hao and H.J. Maris, Phys. Rev. Lett. 84, 5556 (2000).

${ }^{10}$ N.-W. Pu, J. Bokor, S. Jeong, and R.-A. Zhao, Appl. Phys. Lett. 74, 320 (1999).

${ }^{11}$ D.N. Hill, S.A. Cavill, A.V. Akimov, E.F. Ouali, E.S. Moskalenko, L.J. Challis, A.J. Kent, F.W. Sheard, P. Král, and
M. Henini, Phys. Status Solidi B 204, 431 (1997).

${ }^{12}$ X. Hu and F. Nori, Phys. Rev. Lett. 76, 2294 (1996).

${ }^{13}$ C.-K. Sun, J.-C. Liang, C.J. Stanton, A. Abare, L. Coldren, and S.P. DenBaars, Appl. Phys. Lett. 75, 1249 (1999).

${ }^{14}$ C.-K. Sun, J.-C. Liang, and X.-Y. Yu, Phys. Rev. Lett. 84, 179 (2000).

${ }^{15}$ C.-K. Sun, Y.-K. Huang, J.-C. Liang, A. Abare, and S.P. DenBaars, Appl. Phys. Lett. 78, 1201 (2001).

${ }^{16}$ Ü. Özgür, C.-W. Lee, and H.O. Everitt, Phys. Rev. Lett. 86, 5604 (2001).

${ }^{17}$ L. Challis, Nature (London) 367, 687 (1994).

${ }^{18}$ A. Bartels, T. Dekorsy, H. Kurz, and K. Köhler, Appl. Phys. Lett. 72, 2844 (1998).

${ }^{19}$ A. Arbouet, N. Del Fatti, and F. Vallée (to be published).

${ }^{20}$ G.D. Sanders, C.J. Stanton, and C.S. Kim, Phys. Rev. B 64, 235316 (2001).

${ }^{21}$ G.-W. Chern and C.-K. Sun, Phys. Rev. B (to be published).

${ }^{22}$ S.F. Chichibu, A.C. Abare, M.S. Minsky, S. Keller, S.B. Fleischer, J.E. Bowers, E. Hu, U.K. Mishra, L.A. Coldren, S.P. DenBaars, and T. Sota, Appl. Phys. Lett. 73, 2006 (1998).

${ }^{23}$ A.V. Kuznetsov and C.J. Stanton, Phys. Rev. B 51, 7555 (1995).

${ }^{24}$ D.A.B. Miller, D.S. Chemla, and S. Schmitt-Rink, Phys. Rev. B 33, 6976 (1986). 